\title{
Influence of the actions observed on cervical motion in patients with chronic neck pain: a pilot study
}

\author{
Lucía de-la-Puente-Ranea ${ }^{1,2}$, Beatriz García-Calvo ${ }^{3}$, Roy La Touche ${ }^{1,24,5}$, Josué Fernández-Carner0 ${ }^{1,24,6}$, Alfonso Gil-Martínez 1,2,5,** \\ ${ }^{1}$ Centro Superior de Estudios Universitarios La Salle, Universidad Autónoma de Madrid, Faculty of Health Science, Department of Physiotherapy, Aravaca, Madrid, Spain \\ ${ }^{2}$ Hospital La Paz Institute for Health Research, IdiPAZ, Madrid, Spain \\ ${ }^{3}$ Ruber International Hospital, Madrid, Spain \\ ${ }^{4}$ Research Group on Movement and Behavioral Science and Study of Pain, The Center for Advanced Studies University La Salle, Universidad Autónoma de Madrid, Madrid, \\ Spain \\ ${ }^{5}$ Institute of Neuroscience and Craniofacial Pain (INDCRAN), Madrid, Spain \\ ${ }^{6}$ Universidad Rey Juan Carlos, Faculty of Health Science, Department of Physical Therapy, Alcorcón, Madrid, Spain
}

The aim of the present pilot study was to prove if the action-observation (AOb) improved the cervical range of motion (CROM) in patients with nonspecific chronic neck pain (CNP). Double blind pilot study. A total of 28 subjects were randomly assigned to an effective-movement group $(n=14)$ and an ineffective-movement group $(n=14)$. The follow-up consisted of: pretreatment, posttreatment and 10 min after second measurement (motor imagery). Outcome measures were CROM, and pressure pain detection thresholds (PPDTs). No statistical differences were found in baseline on CROM and on the PPDT. Test for independent groups revealed significant changes in cervical rotation movement. Both groups in posttreatment $(P=0.042$; Cohen $d=0.81)$ and after $10 \mathrm{~min}$
$(P=0.019$; Cohen $d=0.9)$. For intragroup PPDT, the Wilcoxon test revealed significant effects in the effective movement at $\mathrm{C} 2$ of the pre to 10-min post $(P=0.040)$. However, the ineffective movement revealed a significant reduction in PPDT in zygapophyseal joint of C5-C6 as the pre to $\operatorname{post}(P=0.010)$ as the pre to 10 -min post $(P=0.041)$ periods. In conclusions this pilot study demonstrated that the effective $\mathrm{AOb}$ produced significant changes versus ineffective $A O b$ in the CROM and it could influences in PPT in subject with CNP immediately.

Keywords: Observed action, Chronic neck pain, Mirror neuron

\section{INTRODUCTION}

Chronic neck pain (CNP) is one of the most common causes of pain and disability. The prevalence of recurring CNP is approximately $15 \%$ in the adult population (Falco et al., 2012). The condition increases disability, significantly reduces quality of life and has a considerable socioeconomic impact (Falco et al., 2012; Izquierdo Pérez et al., 2014; Serrano-Aguilar et al., 2011; Soysal et al., 2013). Moreover, CNP increases work absenteeism and the consumption of medicinal products (Vonk et al., 2009). Neck disability decreases the daily physical activity of these patients and is one of the factors that perpetuate its chronicity (Soysal et al., 2013).

Nonspecific neck pain has no particular origin (Serrano-Aguilar et al., 2011) but is thought to result from joint and muscle structures (Monticone et al., 2012; Serrano-Aguilar et al., 2011). It has also been observed that atlantoaxial and zygapophyseal joints and the intervertebral cervical discs are involved in generating pain (Falco et al., 2012), although numerous authors consider it a multifactorial disease (Monticone et al., 2012). The main contributing factors include age, poor posture, repetitive stress, and social and psychosocial factors, which can increase disability, stress and anxiety (Monticone et al., 2012).

Certain patients with chronic pain show signs of central sensitization and abnormal pain modulation, causing changes in the peripheral somatosensory function, the spinal cord and cerebral cortex (Marcuzzi et al., 2013). Kong et al. (2013) showed that there are
*Corresponding author: Alfonso Gil-Martínez (iD http://orcid.org/0000-0003-0115-088X Hospital Universitario La Paz, Unidad de apoyo a la Investigación en Enfermería. Planta 1a Hospital Maternal, Paseo de la Castellana, 261. 28046, Madrid, Spain Tel: +34-666137908, Fax: +34-913571730, E-mail: fongilmar@gmail.com Received: May 6, 2016 / Accepted: July 14, 2016
This is an Open Access article distributed under the terms of the Creative Commons Attribution Non-Commercial License (http://creativecommons.org/licenses/by-nc/4.0/) which permits unrestricted non-commercial use, distribution, and reproduction in any medium, provided the original work is properly cited. 
cortical changes in the primary somatosensory area (S1) in patients with nonspecific chronic back pain (Kong et al., 2013). S1 is activated during the presentation of harmful stimuli, although it is thought that $\mathrm{S} 1$ also modulates the location and intensity of the pain and is an area with significant neuroplasticity (Kong et al., 2013). These authors also demonstrated a negative correlation between pain intensity and brain connectivity, such that patients with greater pain had reduced functional connectivity (Kong et al., 2013).

A number of authors have demonstrated that if a somatosensory disorder is generated (by performing a specific task) then this will directly cause changes in motor learning due to their interrelation (Vidoni et al., 2010). These changes in the central nervous system can affect the pain intensity, the pressure pain threshold and the reported pain (Staud, 2011).

Currently, we know that chronic pain can last far longer than the initial disease and that social and psychological factors can influence its perpetuation. Thus, the learning of new experiences can modify the acquired pathological behaviors, thereby decreasing the perception of pain and the disability (Vonk et al., 2009).

The observation of activities can produce changes in the representation of the motor cortex (Cantarero et al., 2011), given that observation reinforces the cortical representation of the action (Ertelt et al., 2012). Mirelman et al. (2010) demonstrated that action-observation $(\mathrm{AOb})$ therapy improves strength and functionality in patients with chronic disease (Mirelman et al., 2010).

It has been suggested that visual training creates the necessary conditions for functional recovery based on the mirror-neuron system (Prochnow et al., 2013). The mirror neurons are a specific class of neurons that are activated both in the execution of a movement itself and in the observation of the same or similar motion executed by another individual (Sale and Franceschini, 2012). Several studies have shown that this specific neuronal network is activated by learning the movement through motor execution, observation, imitation, or imagination (Sale and Franceschini, 2012; Villiger et al., 2013).

This neuronal system is found primarily in the inferior parietal lobule and in the inferior frontal gyrus (Nedelko et al., 2010) but is also found in the primary motor cortex, secondary motor cortex, premotor cortex, and supplementary motor area (Prochnow et al., 2013; Sale and Franceschini, 2012).

Recent studies have shown that the observation of an action increases corticospinal excitability in healthy adults when combined with the intent to imitate and with physical practice (Roosink and Zijdewind, 2010; Sale and Franceschini, 2012; Stefan et al., 2008). Other studies have asserted that repeated observation of a movement model is effective for improving the performance of this motor ability (Hodges et al., 2007; Ray et al., 2013; Vogt and Thomaschke, 2007). The process that causes this change is still unknown, but there is evidence that the premotor cortex and the inferior parietal lobule are activated by observing or performing the same movement (Ray et al., 2013).

There are two lines of research that show the hypothetical relationship between the activation of the motor system during $\mathrm{AOb}$ therapy and the adaptation of this system after repeating the $\mathrm{AOb}$ (Ray et al., 2013): (a) there is increased activity of the corticospinal system during observation (Fadiga et al., 1995; Ray et al., 2013) and (b) there are changes in the primary motor cortex after observing a repeated action (Ray et al., 2013; Stefan et al., 2008). Ray et al. (2013) demonstrated that there is a positive correlation between the amount of change in the cortical representation of the movement and the degree of corticospinal activation during the observation of a repeated action.

The main objective of this pilot study was to determine whether intervention through observed activities improves the cervical range of motion (CROM) in patients with nonspecific CNP. The secondary objective was to determine the effect of the intervention on the pressure pain detection threshold (PPDT) in these patients.

\section{MATERIALS AND METHODS}

To ensure the methodological quality of the study, as well as to adapt to the international criteria for presenting clinical reports, we followed the points of the CONSORT list (Schulz et al., 2010).

\section{Design}

The study was a double-blind, randomized pilot study. The randomization was performed by an individual external to the study, prior to the start of the study, using GraphPad QuickCalcs (GraphPad Software Inc., La Jolla, CA, USA). The intervention group was hidden from the blinded evaluator using an opaque envelope. The evaluator was thus unaware at all times as to which group the next patient belonged to. The study was approved by the Ethics Committee of La Paz University Hospital (HULP) (Code 4054). All patients completed the informed consent process before starting the study.

\section{Selection criteria}

Patients with CNP between the ages of 18 and $60 \mathrm{yr}$ were recruited for the study, which was conducted in the Functional Rehabilitation Institute of the La Salle Centre for University Studies 
and in the HULP Physical Therapy Unit from October 2013 to June 2014.

The selection criteria were as follows: nonspecific CNP and between 18 and $60 \mathrm{yr}$ of age. Exclusion criteria were cervical osteoarthritis or polyarthrosis, rheumatic disease, history of cervical hernia, whiplash syndrome, surgery on the neck, face or shoulders, systemic disease, medical history of cancer, significant trauma that the patient relates to their pain and/or refusal to participate. Once the candidate's suitability for the study had been checked, the participant was issued a number in ascending order and randomly assigned to one of the treatment groups.

\section{Intervention}

The patients were divided into two groups, an effective movement group and an ineffective movement group. Each patient was assigned a number for performing the blinding. The intervention consisted of a single session during which three measurements were performed: pretreatment, posttreatment and $10 \mathrm{~min}$ after the second measurement.

\section{Effective movement group}

The participants assigned to this group were shown a 1-min video showing an individual performing a cervical rotation movement to each side, first 10 times towards the right and then 10 times towards the left. The movement was performed effectively over the entire course of the cervical path, approximately $80^{\circ}$.

\section{Ineffective movement group}

The participants assigned to this group were shown a 1-min video showing an individual performing a cervical rotation movement ineffectively, without achieving the maximum cervical travel, performing approximately $40^{\circ}$. First, 10 movements towards the right were performed and then 10 towards the left.

The randomization was performed in a hidden manner, so that the evaluator knew the number corresponding to each subject but not the treatment group to which they belonged.

\section{Double-blind}

To perform the blinding of the evaluator, a study assistant played the video assigned to each subject without the evaluator being present. After the video presentation, the evaluator returned to the treatment room to complete the subsequent and 10-min measurements.

The patient blinding consisted of not having the patient aware at any time as to whether the video they observed was the effective or ineffective movement. To facilitate the blinding, the individual appearing in the videos was the same model throughout and showed no indication of pain or disability, and the video was played in silence for all of the patients. This blinding was checked upon completion of the trial by asking the patients which video they thought they had observed, to which they replied that they had only watched a video of an individual moving their neck without specifying anything more.

Significant efforts were undertaken to avoid biases in the study. In addition to conducting double-blinding of the evaluator and participants, the treatment was always performed in the same room, measuring the confounding variables to control the sample and using the same material. The order in which the measurements were performed was consistent, as was the time during which the participants had to think about the action that they had observed.

\section{Description of the outcome variables}

After each patient had given their informed consent to participate in the pilot study, they were given a battery of questionnaires to be filled out on the first day of measurement. These included several self-reports on psychological variables and a questionnaire on demographic variables that collected information on age, weight, height, medication, and pain chronicity and intensity. We assessed the psychological variables using the self-reported measurements. Catastrophism was assessed with the version of the Pain Catastrophizing Scale translated and validated in Spanish (García Campayo et al., 2008); fear of movement was measured with the Spanish version of the Tampa Scale for Kinesiophobia (TSK-11) (Gómez-Pérez et al., 2011); fear avoidance beliefs were assessed with the Spanish version of the Fear Avoidance Belief Questionnaire (Kovacs et al., 2006); depressive symptoms were assessed with the Beck Depression Inventory; anxiety was assessed with the Spanish version of State-Trait Anxiety Inventory (Perpiñá-Galvañ et al., 2013); and neck disability was assessed with the Spanish version of the Neck Disability Index (Andrade Ortega et al., 2010).

\section{Cervical joint range of motion}

To quantify this movement, we used a CROM tool validated by Audette et al. (2010) that assessed the active movement of the cervical spine. The patient was seated in a chair, and the goniometer was placed on their head. The patient was then asked to perform the maximum flexoextension movement, lateral inclinations and rotations. Each movement was recorded twice; if the difference exceeded $5^{\circ}$, a third measurement was conducted. 


\section{Pressure pain detection threshold}

This was defined as the minimal amount of pressure that the patient perceived as painful. The tool used was an algometer (model Fx. 25 Force Gage, Wagner Instruments, Greenwich, CT, USA), which consists of a $1-\mathrm{cm}^{2}$ rubber tip attached to a pressure meter. The data were collected in $\mathrm{kg} / \mathrm{cm}^{2}$, with a 30-sec interval between each measurement, thereby avoiding the temporal summation of stimuli. This apparatus is a highly effective and clinically valid instrument for this purpose (Kinser et al., 2009).

\section{Data analysis}

The IBM SPSS Statistics ver. 20.0 (IBM Co., Armonk, NY, USA) was employed for the data analysis. We used the ShapiroWilk test to determine the normal data distribution. To compare the means of the intergroup and intragroup CROM, we used the $t$-test for independent and related samples. For the comparison of the PPDT, we performed the Wilcoxon test for related samples and the Mann-Whitney $U$-test for independent samples. For all statistical comparisons, we used $P<0.05$ as the criterion of significance, with a 95\% confidence interval. Effect size was calculated through Cohen $\mathrm{d}$ for parametric variables as:

$$
d=\frac{\left|\overline{x_{1}}-\bar{x}_{2}\right|}{\sqrt{\frac{S_{1}^{2}+S_{2}^{2}}{2}}}
$$

\section{RESULTS}

\section{Participants}

The patients were recruited from February 2014 to May 2014. A total of 30 patients were recruited, two of whom were excluded for meeting one of the exclusion criteria. Twenty-eight patients with nonspecific CNP were selected for the pilot study; 14 were

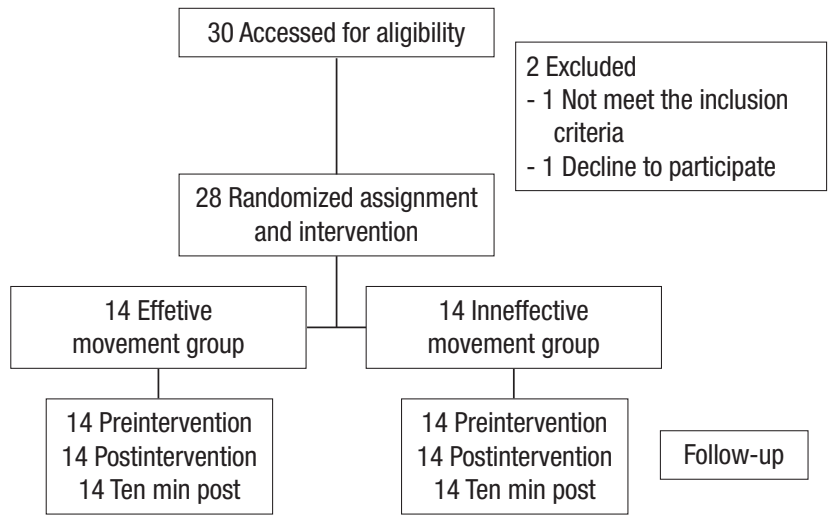

Fig. 1. Flow diagram of the clinical trial. randomly assigned to the effective movement group and 14 were assigned to the ineffective movement group (Fig. 1). All variables assessed followed a normal distribution $(P>0.05)$, starting from a homogeneous sample, except for the PPDT values. There were no statistically significant differences in the demographic characteristics except for pain chronicity (Table 1). Moreover, there were no statistically significant differences in the psychological control variables, except in the TSK-11 within the effective movement group (Table 2).

\section{Intervention}

\section{Cervical joint range of movement}

In terms of the intragroup analysis, significant changes only occurred in the effective movement group from the posttreatment to the 10-min measurement in both flexion $(P=0.020)$ and extension $(P=0.025)$. The $t$-test for independent samples was per-

Table 1. Demographic characteristics and baseline measures

\begin{tabular}{lcc} 
Characteristic & $\begin{array}{c}\text { Effective movement } \\
\text { group }(\mathrm{n}=14)\end{array}$ & $\begin{array}{c}\text { Ineffective movement } \\
\text { group }(\mathrm{n}=14)\end{array}$ \\
\hline Age $(\mathrm{yr})$ & $29.50 \pm 9.66$ & $29.86 \pm 12.89$ \\
Sex, male:female & $5(35.7): 9(64.3)$ & $2(14.3): 12(85.7)$ \\
Medication, yes:no & $2(14.3): 12(85.7)$ & $6(42.9): 8(57.1)$ \\
Height $(\mathrm{cm})$ & $172.00 \pm 9.58$ & $162.64 \pm 7.28$ \\
Weight $(\mathrm{kg})$ & $62.00 \pm 12.11$ & $60.68 \pm 8.75$ \\
Body Mass Index $\left(\mathrm{kg} / \mathrm{m}^{2}\right)$ & $20.76 \pm 2.06$ & $23.05 \pm 3.87$ \\
Chronicity $(\mathrm{yr})$ & $6.25 \pm 5.05^{*}$ & $4.86 \pm 4.00^{*}$ \\
Days of pain $(\mathrm{mo})$ & $20.71 \pm 10.19$ & $22.86 \pm 7.47$ \\
Pain intensity & $18.00 \pm 14.92$ & $26.50 \pm 16.71$ \\
\hline
\end{tabular}

Values are presented as mean \pm standard deviation or number $(\%)$. ${ }^{*} P<0.05$.

Table 2. Baseline characteristics of psychological variables

\begin{tabular}{lcc}
\hline Variable & $\begin{array}{c}\text { Effective movement } \\
\text { group }(n=14)\end{array}$ & $\begin{array}{c}\text { Ineffective movement } \\
\text { group }(n=14)\end{array}$ \\
\hline FABO & $27.29 \pm 10.56$ & $39.21 \pm 15.10$ \\
PCS & $12.64 \pm 8.85$ & $12.86 \pm 7.50$ \\
BDI & $8.38 \pm 3.95$ & $9.36 \pm 4.91$ \\
NDI & $8.71 \pm 4.25$ & $12.00 \pm 4.72$ \\
S-ANXIETY & $23.50 \pm 4.93$ & $23.29 \pm 4.45$ \\
T-ANXIETY & $26.00 \pm 5.99$ & $25.64 \pm 4.50$ \\
TSK-11 & $22.57 \pm 5.39 *$ & $24.43 \pm 5.92$
\end{tabular}

Values are presented as mean \pm standard deviation.

FABQ, Fear Avoidance Belief Questionnaire; PSC, Pain Catastrophizing Scale; BDI, Beck Depression Inventory; NDI, Neck Disability Index; S-ANXIETY, State Anxiety Inventory; T-ANXIETY, Trait Anxiety Inventory; TSK-11, Tampa Scale for Kinesiophobia short version.

${ }^{*} P<0.05$ 
formed on the difference in means between the groups in cervical rotations and no statistically significant changes were found $(P=$ 0.069).

The intergroup analysis observed significant changes in the cervical rotation movement, both at posttreatment $(P=0.042$; Cohen $d=0.81)$ and at 10 minutes $(P=0.019$; Cohen $d=0.9)$ (Table 3).

\section{Pressure pain detection threshold}

There were no statistically significant changes in the intergroup data. Within the intragroup results, statistically significant results were obtained for the effective movement group in $\mathrm{C} 2$ joints by obtaining the median and interquartile range, between the pretreatment and 10-min posttreatment (pre, 0.610 [0.268-1.345];

Table 3. Differences between groups for changes from baseline in patients range of motion score

\begin{tabular}{llccc}
\hline Range & Follow-up & Effective movement group & Ineffective movement group & $P$-value \\
\hline Flexion & Pre & $56.86 \pm 13.53$ & $51.44 \pm 10.19$ & 0.242 \\
& Post & $56.94 \pm 12.77$ & $51.48 \pm 11.26$ & 0.241 \\
Extension & 10 -min post & $53.46 \pm 13.31$ & $50.51 \pm 7.71$ & 0.481 \\
& Pre & $70.32 \pm 9.21$ & $66.99 \pm 14.87$ & 0.483 \\
& Post & $72.36 \pm 9.99$ & $65.21 \pm 14.37$ & 0.139 \\
Lateral flex & 10 -min post & $69.93 \pm 9.38$ & $63.57 \pm 15.61$ & 0.203 \\
& Pre & $84.21 \pm 17.66$ & $84.19 \pm 15.43$ & 0.997 \\
Rotation & Post & $85.39 \pm 16.04$ & $80.71 \pm 12.78$ & 0.401 \\
& 10-min post & $83.58 \pm 19.65$ & $80.65 \pm 15.35$ & 0.664 \\
& Pre & $133.09 \pm 16.41$ & $125.15 \pm 17.89$ & 0.231 \\
& Post & $136.58 \pm 18.18$ & $123.00 \pm 15.26$ & $0.042^{*}$ \\
\hline
\end{tabular}

Values are presented as mean \pm standard deviation.

${ }^{*} P<0.05$.

Table 4. Differences between and within groups for changes from baseline in patients pressure pain thresholds

\begin{tabular}{|c|c|c|c|c|c|c|}
\hline \multirow{2}{*}{ Measurement } & \multirow{2}{*}{ Pre } & \multirow{2}{*}{ Post } & \multirow{2}{*}{ 10-min Post } & \multicolumn{3}{|c|}{ Wilcoxon test ( $P$-value) } \\
\hline & & & & Pre vs Post & Post vs 10-min Post & Pre vs 10-min Post \\
\hline \multicolumn{7}{|l|}{ Suboccipital } \\
\hline Group A & $0.643(0.335-1.176)$ & $0.575(0.304-1.108)$ & $0.673(0.277-1.163)$ & 0.300 & 0.109 & 0.683 \\
\hline Group B & $0.528(0.318-0.895)$ & $0.433(0.220-0.924)$ & $0.398(0.243-0.690)$ & 0.140 & 0.198 & 0.056 \\
\hline Mann-Whitney U-test & 0.491 & 0.476 & 0.190 & & & \\
\hline \multicolumn{7}{|l|}{$\mathrm{C} 2$} \\
\hline Group A & $0.610(0.268-1.345)$ & 0.695 (0.258-1.483) & 0.605 (0.245-1.393) & 0.753 & 0.315 & $0.040^{*}$ \\
\hline Group B & $0.525(0.238-0.790)$ & $0.385(0.215-0.698)$ & $0.385(0.200-0.643)$ & 0.142 & 0.975 & 0.069 \\
\hline Mann-Whitney U-test & 0.323 & 0.161 & 0.148 & & & \\
\hline \multicolumn{7}{|l|}{ C5-C6 } \\
\hline Group A & $0.630(0.318-1.144)$ & 0.701 (0.269-1.521) & 0.673 (0.230-1.281) & 0.443 & 0.807 & 0.506 \\
\hline Group B & $0.650(0.274-1.099)$ & $0.428(0.248-0.661)$ & $0.405(0.291-0.824)$ & $0.010^{*}$ & 0.362 & $0.041^{*}$ \\
\hline Mann-Whitney U-test & 0.198 & 0.301 & 0.370 & & & \\
\hline \multicolumn{7}{|l|}{ Upper trapezius } \\
\hline Group A & $0.593(0.370-1.221)$ & 0.663 (0.295-1.203) & 0.693 (0.286-1.096) & 0.615 & 0.950 & 0.875 \\
\hline Group B & $0.638(0.351-0.730)$ & $0.603(0.260-0.878)$ & $0.505(0.265-0.925)$ & 0.683 & 0.675 & 0.875 \\
\hline Mann-Whitney U-test & 0.550 & 0.550 & 0.520 & & & \\
\hline \multicolumn{7}{|l|}{ Phalange } \\
\hline Group A & $0.830(0.398-1.401)$ & 0.740 (0.463-1.255) & 0.970 (0.425-1.535) & 0.329 & 0.315 & 0.507 \\
\hline Group B & $0.760(0.440-1.450)$ & 0.795 (0.373-1.745) & $0.765(0.370-1.320)$ & 0.925 & 0.272 & 0.433 \\
\hline Mann-Whitney U-test & 0.854 & 0.730 & 0.629 & & & \\
\hline
\end{tabular}

Values are presented as median (range).

Group A, effective movement group; Group B, ineffective movement group.

${ }^{*} P<0.05$. 
10-min post, 0.605 [0.245-1.393]; $P=0.04)$, with greater hyperalgesia. In contrast, there were significant changes in the ineffective movement group for C5-C6 zygapophyseal joints for both pretreatment to posttreatment (pre, 0.650 [0.274-1.099]; post, 0.428 [0.248-0.661]; $P=0.010$ ) and for pretreatment to 10 -min posttreatment (pre, 0.650 [0.274-1.099]; 10-min post, 0.405 [0.291-0.824]; $P=0.041$ ), with greater hyperalgesia (Table 4).

\section{DISCUSSION}

The principal hypothesis was that intervention with image observation therapy improves the CROM and produces a hypoalgesic effect in participants. Our results partially prove this hypothesis due to the observed increase in the cervical range of rotation and the increase in PPDT on a point in the upper cervical region after the intervention. However, we have insufficient scientific evidence to perform a theoretical comparison with our results. However, a study similar to ours performed by Nobusako et al. (2012) proposed an experiment for the observation and recognition of a cervical rotation motor task. The results showed that the intervention improved the CROM and decreased the pain intensity (Nobusako et al., 2012). All of the results obtained in this research will be discussed in depth below.

\section{Cervical range of motion}

Based on the results, we can confirm that effective $\mathrm{AOb}$ immediately improves the CROM in patients with nonspecific CNP. In our study, we obtained differences of $7^{\circ}$ between the groups in the entire movement, with $6.1^{\circ}$ being the minimum detectable change for the right rotation and $4.9^{\circ}$ for the left rotation, as reported by Audette et al. (2010). This change is produced in both the visualization of an $\mathrm{AOb}$ and the thinking process conducted by the patient.

Most studies do not interpret $\mathrm{AOb}$ as the only element influencing the participant but rather are directed towards demonstrating the neural processes that are generated. Stanley and Miall, (2007) represented congruent and incongruent sequential images of movements of the right hand. The participants had to observe the visual sequence while simultaneously performing the movement with their right hand. The study achieved greater activation of the upper parietal lobe and primary visual cortex for the participants who watched the visual series and hand movements that were in disagreement than those who watched the sequential congruent version (Stanley and Miall, 2007). The authors showed that when there is a change in the relationship between the action performed and the images observed there is increased activation of the early visual areas (Stanley and Miall, 2007). This demonstration leads us to assume that the activation of the parietal areas and visual cortex are related to the $\mathrm{AOb}$ and the movement performed, serving as a basis for our study.

Moreover, Bortoletto et al., (2013) showed that motor-visual interference is modulated through higher cognitive processes, in such a way that when we pay attention to our own actions, the actions performed by others have less interference than our own (Bortoletto et al., 2013). This leads to the possibility that the influence of our video could have been lower due to the fact that the therapy conducted was not accompanied by the participant's own movement.

Lastly, this type of treatment has also been used on patients with neurological involvement. Celnik et al., (2008) showed that AOb therapy improved motor training and motor memory in patients with chronic stroke (Celnik et al., 2008). Thus AOb might create cortical changes and improve motor skills.

One of the researchers' main theories is that CROM is influenced by $\mathrm{AOb}$ thanks to the activation of the mirror-neuron system. A considerable number of neuroimaging studies have shown that this neural network is located in the ventral and dorsal areas of the premotor cortex, in the inferior frontal gyrus, in the temporal visual and posterior parietal regions and in the somatosensory cortex (Caspers et al., 2010; Keysers et al., 2010; Vogt et al., 2013).

The motor system is boosted due to 2 basic processes that are generated in AOb: the initial recognition of the action (through the temporoparietal circuit) and motor simulation (through the parietofrontal circuit) (Kilner, 2011; Vogt et al., 2013). Thus, motor simulation is useful when the observer must predict the $\mathrm{AOb}$ reference point in order to adjust their own action towards this reference point or to synchronize their own action (Vogt et al., 2013). Borroni et al. (2005), studied the relationship between the motor system and AOb through cortical excitability. Their results indicated that the excitability of the motor system has a cyclic rhythm similar to that of $\mathrm{AOb}$, which suggests a close relationship between the two processes.

If we analyze the results in more detail, the videos of the effective and ineffective movements produced an increase of $3^{\circ}$ and a decrease of $4^{\circ}$ in cervical rotation, respectively. This outcome suggests that observing an image of a movement conducted incorrectly creates maladaptive changes in these patients. This situation could be explained through two possible theories. On one hand, the presence of areas related to pain, called pain matrices, has been widely related to cognitive and memory processes. Chronic pain 
creates cortical dysfunctions in these areas, which might mean that the ineffective video is analyzed erroneously, creating a reduction in the movement (Hsu et al., 2009; Kuchinad et al., 2007). Another hypothesis is related to the change in psychological aspects that affect patients with chronic pain. Firstly, catastrophism is associated with increased activity in areas related to motor control (Gracely et al., 2004). Secondly, within the fear of movement variable, significant differences were obtained between the two groups, with greater fear in the ineffective movement group. This result suggests that the CROM results in this group were lower than after the intervention due to the interference of fear of movement. It should be noted that the fear of movement variable measured with the TSK-11 had differences between the two groups, with fear being greater in the ineffective movement group, suggesting that the results of the CROM in this group were lower after the intervention due to the interference of fear of movement. Current evidence related to CNP has shown that fear of movement is related to CROM and to the level of disability of this type of patient (Sarig Bahat et al., 2014).

This study analyzed the influence of $\mathrm{AOb}$ regarding the increase in joint range. There is considerable evidence of the cortical activation produced by this therapy; more studies are therefore needed to define the medium to long-term effects of $\mathrm{AOb}$ and that integrate this treatment in a motor training protocol, given that other types of mental practices such as motor imagery in combination with physical practice have been shown effective in improving joint range of movement (Guillot et al., 2010; Williams et al., 2004).

Patients with chronic pain have altered somatosensory imagery areas of the brain, which causes a loss of representational areas and sensory impairment, triggering a distorted body image.

Tissue pain limits the range of movement of patients with neck pain, which when prolonged can trigger compensatory movements to reduce the pain, such as muscle contractions that increase the vicious cycle of cortical impairment of motor processing. Thus, the observation of a complete movement can produce neural motor images and trigger the specific motor cortex areas of the neck (Buccino et al., 2001).

One of the positive aspects of $\mathrm{AOb}$ is the minimum risk for the patient. Manual therapy, specifically high-velocity cervical techniques, causes effects on CROM similar to those obtained by this study but has a high risk of injuring the patient. This suggests that the use of $\mathrm{AOb}$ is an effective alternative for this type of high-velocity cervical technique.

\section{Pressure pain detection threshold}

$\mathrm{AOb}$ affects the PPDT of patients with nonspecific CNP. It generates changes in $\mathrm{C} 2$, in the effective movement group (hypoalgesia), and in $\mathrm{C5}-\mathrm{C} 6$ when visualizing the ineffective movement (hyperalgesia).

Once the effective movement is observed, the PPDT in C2 increased. Subsequent to the integration of the video, the process was conducted in reverse, decreasing the threshold towards initial parameters. This change can be explained through the observation of the video, where the atlantoaxial joint complex is designed to perform the maximum rotation in the horizontal plane, allowing for approximately $45^{\circ}$ of axial rotation in both directions (Neumann, 2007). This is related to the increase in PPDT through AOb.

Moreover, when the participant observed the incorrect movement, the PPDT decreased and remained that way after the process of integration. This fact suggests that the observation of an incomplete movement produced greater negative effects than viewing a correct image.

Most current studies have investigated the cortical effects produced by $\mathrm{AOb}$ associated with the integration of a painful stimulus. Forkmann et al. (2013) explored the neural mechanisms that generate pain in the coding of visual stimuli (Forkmann et al., 2013). The authors found that painful stimulation decreased performance in the visual coding of a surprise task, combined with reduced functional connectivity of the hypothalamus (Forkmann et al., 2013). These results suggest that patients with chronic pain have a reduced capacity for reaction when faced with new stimuli. Park et al. (2001) confirmed these results in patients with fibromyalgia. However, our results showed for the first time how $\mathrm{AOb}$ influences the PPDT without the support of a motor activity.

More studies are needed to interpret the effect of $\mathrm{AOb}$ on the PPDT in patients with CNP.

\section{Limitations of the study}

There are limitations to consider when interpreting the results of this study. Firstly, the data obtained at this research are preliminary outcomes and they are useful to calculate the sample size for future research. In order to extrapolate accurate and representative results of a future clinical trial the sample size should be higher. Secondly, there is scarce literature related to this subject for designing the study. However, we believe that the methodology was developed correctly based on methodological models that have reported similar therapies. Thirdly, the video consists of only the observation of cervical rotation; we therefore cannot extend these results to the other cervical movement planes. Fourth, there was 
no short-term follow-up. More studies are needed to investigate this therapy in the medium to long term. Lastly, the therapy was limited to participants with nonspecific CNP; it is therefore difficult to generalize the results to other populations that differ from this group.

This pilot study reveals that effective AOb improves the CROM in patients with nonspecific CNP in a short period of time. Moreover, $\mathrm{AOb}$ creates a significant effect on the PPDT. However, more studies are necessary to investigate the influence of an observed ineffective action on these patients and that show whether this therapy is beneficial in a motor training protocol in the medium to long term.

\section{CONFLICT OF INTEREST}

No potential conflict of interest relevant to this article was reported.

\section{REFERENCES}

Andrade Ortega JA, Delgado Martínez AD, Almécija Ruiz R. Validation of the Spanish version of the Neck Disability Index. Spine (Phila Pa 1976) 2010;35:E114-118.

Audette I, Dumas JP, Côté JN, De Serres SJ. Validity and between-day reliability of the cervical range of motion (CROM) device. J Orthop Sports Phys Ther 2010;40:318-323.

Borroni P, Montagna M, Cerri G, Baldissera F. Cyclic time course of motor excitability modulation during the observation of a cyclic hand movement. Brain Res 2005;1065:115-124.

Bortoletto M, Mattingley JB, Cunnington R. Effects of context on visuomotor interference depends on the perspective of observed actions. PLoS One 2013;8:e53248.

Buccino G, Binkofski F, Fink GR, Fadiga L, Fogassi L, Gallese V, Seitz RJ, Zilles K, Rizzolatti G, Freund HJ. Action observation activates premotor and parietal areas in a somatotopic manner: an fMRI study. Eur J Neurosci 2001;13:400-404.

Cantarero G, Galea JM, Ajagbe L, Salas R, Willis J, Celnik P. Disrupting the ventral premotor cortex interferes with the contribution of action observation to use-dependent plasticity. J Cogn Neurosci 2011;23: 3757-3766.

Caspers S, Zilles K, Laird AR, Eickhoff SB. ALE meta-analysis of action observation and imitation in the human brain. Neuroimage 2010; 50:1148-1167.

Celnik P, Webster B, Glasser DM, Cohen LG. Effects of action observation on physical training after stroke. Stroke 2008;39:1814-1820.
Ertelt D, Hemmelmann C, Dettmers C, Ziegler A, Binkofski F. Observation and execution of upper-limb movements as a tool for rehabilitation of motor deficits in paretic stroke patients: protocol of a randomized clinical trial. BMC Neurol 2012;12:42.

Fadiga L, Fogassi L, Pavesi G, Rizzolatti G. Motor facilitation during action observation: a magnetic stimulation study. J Neurophysiol 1995;73:2608-2611.

Falco FJ, Manchikanti L, Datta S, Wargo BW, Geffert S, Bryce DA, Atluri S, Singh V, Benyamin RM, Sehgal N, Ward SP, Helm S 2nd, Gupta S, Boswell MV. Systematic review of the therapeutic effectiveness of cervical facet joint interventions: an update. Pain Physician 2012;15:E839868.

Forkmann K, Wiech K, Ritter C, Sommer T, Rose M, Bingel U. Pain-specific modulation of hippocampal activity and functional connectivity during visual encoding. J Neurosci 2013;33:2571-2581.

García Campayo J, Rodero B, Alda M, Sobradiel N, Montero J, Moreno S. Validation of the Spanish version of the Pain Catastrophizing Scale in fibromyalgia. Med Clin (Barc) 2008;131:487-492.

Gómez-Pérez L, López-Martínez AE, Ruiz-Párraga GT. Psychometric properties of the Spanish version of the Tampa Scale for Kinesiophobia (TSK). J Pain 2011;12:425-435.

Gracely RH, Geisser ME, Giesecke T, Grant MA, Petzke F, Williams DA, Clauw DJ. Pain catastrophizing and neural responses to pain among persons with fibromyalgia. Brain 2004;127(Pt 4):835-843.

Guillot A, Tolleron C, Collet C. Does motor imagery enhance stretching and flexibility? J Sports Sci 2010;28:291-298.

Hodges NJ, Williams AM, Hayes SJ, Breslin G. What is modelled during observational learning? J Sports Sci 2007;25:531-545.

Hsu MC, Harris RE, Sundgren PC, Welsh RC, Fernandes CR, Clauw DJ, Williams DA. No consistent difference in gray matter volume between individuals with fibromyalgia and age-matched healthy subjects when controlling for affective disorder. Pain 2009;143:262-267.

Izquierdo Pérez H, Alonso Perez JL, Gil Martinez A, La Touche R, Lerma-Lara S, Commeaux Gonzalez N, Arribas Perez H, Bishop MD, Fernández-Carnero J. Is one better than another?: A randomized clinical trial of manual therapy for patients with chronic neck pain. Man Ther 2014;19:215-221.

Keysers C, Kaas JH, Gazzola V. Somatosensation in social perception. Nat Rev Neurosci 2010;11:417-428.

Kilner JM. More than one pathway to action understanding. Trends Cogn Sci 2011;15:352-357.

Kinser AM, Sands WA, Stone MH. Reliability and validity of a pressure algometer. J Strength Cond Res 2009;23:312-314.

Kong J, Spaeth RB, Wey HY, Cheetham A, Cook AH, Jensen K, Tan Y, Liu H, Wang D, Loggia ML, Napadow V, Smoller JW, Wasan AD, Gollub 
RL. S1 is associated with chronic low back pain: a functional and structural MRI study. Mol Pain 2013;9:43.

Kovacs FM, Muriel A, Medina JM, Abraira V, Sánchez MD, Jaúregui JO; Spanish Back Pain Research Network. Psychometric characteristics of the Spanish version of the FAB questionnaire. Spine (Phila Pa 1976) 2006;31:104-110.

Kuchinad A, Schweinhardt P, Seminowicz DA, Wood PB, Chizh BA, Bushnell MC. Accelerated brain gray matter loss in fibromyalgia patients: premature aging of the brain? J Neurosci 2007;27:4004-4007.

Marcuzzi A, Dean CM, Hush JM. Early changes in somatosensory function in spinal pain: protocol for a systematic review. Syst Rev 2013;2:90.

Mirelman A, Patritti BL, Bonato P, Deutsch JE. Effects of virtual reality training on gait biomechanics of individuals post-stroke. Gait Posture 2010;31:433-437.

Monticone M, Baiardi P, Vanti C, Ferrari S, Nava T, Montironi C, Rocca B, Foti C, Teli M. Chronic neck pain and treatment of cognitive and behavioural factors: results of a randomised controlled clinical trial. Eur Spine J 2012;21:1558-1566.

Nedelko V, Hassa T, Hamzei F, Weiller C, Binkofski F, Schoenfeld MA, Tüscher O, Dettmers C. Age-independent activation in areas of the mirror neuron system during action observation and action imagery. A fMRI study. Restor Neurol Neurosci 2010;28:737-747.

Neumann DA. Cinesiología del sistema musculoesquelético: fundamentos de la rehabilitación física. Barcelona : Paidotribo Corp.; 2007.

Nobusako S, Matsuo A, Morioka S. Effectiveness of the gaze direction recognition task for chronic neck pain and cervical range of motion: a randomized controlled pilot study. Rehabil Res Pract 2012;2012: 570387.

Park DC, Glass JM, Minear M, Crofford LJ. Cognitive function in fibromyalgia patients. Arthritis Rheum 2001;44:2125-2133.

Perpiñá-Galvañ J, Cabañero-Martínez MJ, Richart-Martínez M. Reliability and validity of shortened state trait anxiety inventory in Spanish patients receiving mechanical ventilation. Am J Crit Care 2013;22:46-52.

Prochnow D, Bermúdez i Badia S, Schmidt J, Duff A, Brunheim S, Kleiser $\mathrm{R}$, Seitz RJ, Verschure PF. A functional magnetic resonance imaging study of visuomotor processing in a virtual reality-based paradigm: Rehabilitation Gaming System. Eur J Neurosci 2013;37:1441-1447.

Ray M, Dewey D, Kooistra L, Welsh TN. The relationship between the motor system activation during action observation and adaptation in the motor system following repeated action observation. Hum Mov Sci 2013;32:400-411.

Roosink M, Zijdewind I. Corticospinal excitability during observation and imagery of simple and complex hand tasks: implications for mo- tor rehabilitation. Behav Brain Res 2010;213:35-41.

Sale P, Franceschini M. Action observation and mirror neuron network: a tool for motor stroke rehabilitation. Eur J Phys Rehabil Med 2012; 48:313-318

Sarig Bahat H, Weiss PL, Sprecher E, Krasovsky A, Laufer Y. Do neck kinematics correlate with pain intensity, neck disability or with fear of motion? Man Ther 2014;19:252-258.

Schulz KF, Altman DG, Moher D; CONSORT Group. CONSORT 2010 statement: updated guidelines for reporting parallel group randomized trials. Ann Intern Med 2010;152:726-732.

Serrano-Aguilar P, Kovacs FM, Cabrera-Hernández JM, Ramos-Goñi JM, García-Pérez L. Avoidable costs of physical treatments for chronic back, neck and shoulder pain within the Spanish National Health Service: a cross-sectional study. BMC Musculoskelet Disord 2011;12:287.

Soysal M, Kara B, Arda MN. Assessment of physical activity in patients with chronic low back or neck pain. Turk Neurosurg 2013;23:75-80.

Stanley J, Miall RC. Functional activation in parieto-premotor and visual areas dependent on congruency between hand movement and visual stimuli during motor-visual priming. Neuroimage 2007;34:290-299.

Staud R. Evidence for shared pain mechanisms in osteoarthritis, low back pain, and fibromyalgia. Curr Rheumatol Rep 2011;13:513-520.

Stefan K, Classen J, Celnik P, Cohen LG. Concurrent action observation modulates practice-induced motor memory formation. Eur J Neurosci 2008;27:730-738.

Vidoni ED, Acerra NE, Dao E, Meehan SK, Boyd LA. Role of the primary somatosensory cortex in motor learning: An rTMS study. Neurobiol Learn Mem 2010;93:532-539.

Villiger M, Estévez N, Hepp-Reymond MC, Kiper D, Kollias SS, Eng K, Hotz-Boendermaker S. Enhanced activation of motor execution networks using action observation combined with imagination of lower limb movements. PLoS One 2013;8:e72403.

Vogt S, Di Rienzo F, Collet C, Collins A, Guillot A. Multiple roles of motor imagery during action observation. Front Hum Neurosci 2013; 7:807.

Vogt S, Thomaschke R. From visuo-motor interactions to imitation learning: behavioural and brain imaging studies. J Sports Sci 2007;25:497517.

Vonk F, Verhagen AP, Twisk JW, Köke AJ, Luiten MW, Koes BW. Effectiveness of a behaviour graded activity program versus conventional exercise for chronic neck pain patients. Eur J Pain 2009;13:533-541.

Williams JG, Odley JL, Callaghan M. Motor imagery boosts proprioceptive neuromuscular facilitation in the attainment and retention of range-of -motion at the hip joint. J Sports Sci Med 2004;3:160-166. 